\title{
Trust and Commitment
}

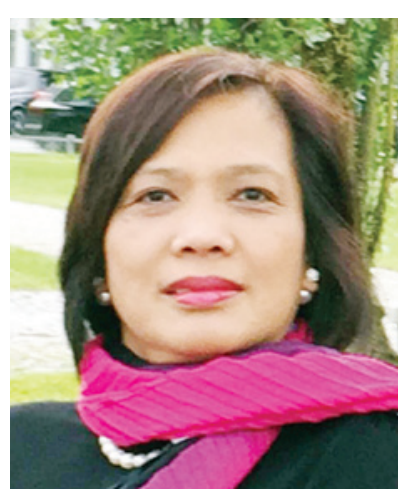

Since November of 2015, the ASEAN Federation of Endocrine Societies (AFES) in its annual meeting has entrusted JAFES to the Philippine team for another term. It is an affirmation of trust that all of us here at the editorial office, on behalf of the PSEDM, keep with the members of the Federation. We stand by our commitment to deliver a world class, high quality publication, and be the voice of endocrinology for Southeast Asia to the global community.

Trust is most important in scientific publications. There is the trust that authors give to the editors. That in their hands, manuscripts are dealt with in accordance with the journal's standards, with utmost conscientiousness, due process, and, more importantly, confidentiality. Precious research ideas are kept within the confines of the agreement that manuscripts never venture outside of scholarly editorial deliberations on their merits (and flaws). Manuscripts are processed always within acceptable turnaround times; although sometimes, due to conflicts in busy schedules, several rounds of searching for the appropriate and reputable reviewers and their actual reviews do take some time.

Authors must be wary of devious publications that solicit articles from researchers, promising questionable index factors, readership and reputation. And it is not impossible for unscrupulous reviewers to delay their reviews of manuscripts intentionally, bringing research ideas from such manuscripts with them, all to the disadvantage of the authors.

On the other hand, editors trust that the authors have abided by the journal's instructions and guidelines. Declarations that the work is original and not for simultaneous consideration elsewhere, attestations of authorship by authors who fulfill the four ICJME criteria, disclosures of any funding and conflicts of interest, are not just forms to sign. They in fact do hold, despite academic pressures and publication requirements in this "publish or perish" world. Submission of the same manuscript to multiple publications is not ethical as it "unnecessarily wastes the precious reviewer resources of one or more journals," to paraphrase a colleague editor-in-chief of another journal.

Duplication and plagiarism are serious offenses that damage trust. Before the age of the Internet, editors relied on the collective knowledge of a pool of trusted reviewers, who, because of their research and publication experience, had the ability to spot plagiarism or another form of authorial misconduct and had the mettle of letting the editors know about this. Currently, as enhancement of quality assurance, editors resort also to antiplagiarism software solutions that compare submitted text with research databases, and flag identical content. While there are open source software options, high quality screening entails significant cost. Starting this year, JAFES is forging a partnership with CrossRef to use CrossCheck.

Institutional ethical approval of research works is essential, and we add the submission of a scanned ethics approval to our Journal's policies.

We view the final published product of JAFES as a testament to the partnership between our valued authors and our esteemed editors, symbolic of the mutual trust between these two parties, who both, in their unique roles, fulfill the commitment of bringing to life scientific information that touches relevant issues of health care in our region.

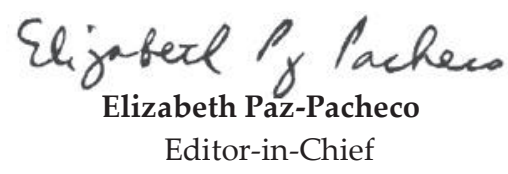

http://dx.doi.org/10.15605/jafes.031.01.01 